\title{
Economía circular, aproximación a un modelo para Pymes exportadoras
}
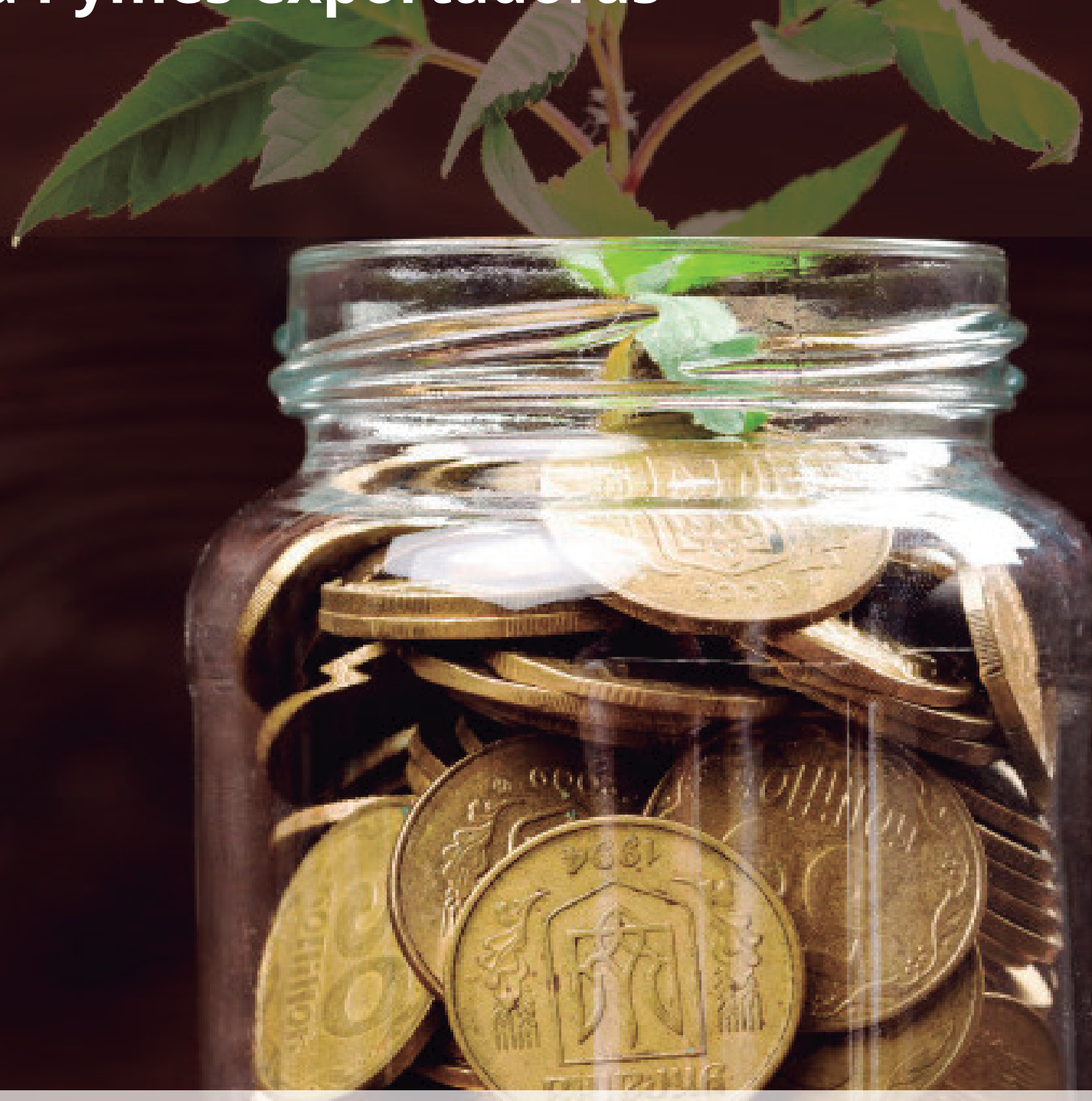

RAFAEL JIMÉNEZ VEGA (

JOHN JAIRO HERNÁNDEZ VILLAMIZAR (1) https://orcid.org/0000-0001-5979-6929 Fundación Universitaria San Mateo / jairohernandezvillamizar@gmail.com

SIRIS MARÍA LÓPEZ RODRÍGUEZ (10) https://orcid.org/0000-0001-7258-6301 Fundación Universitaria San Mateo / dirección.negocios@sanmateo.edu.co

Reconocimiento-SinObraDerivada 4.0 Internacional (CC BY-NC-ND) 
Artículo de investigación

Recepción: 27 de abril de 2020

Aceptación: 04 de agosto de 2020

\section{Cómo citar este artículo}

Jiménez, R., Hernández Villamizar, J., y López Rodríguez, S. (2020).

Economía circular, aproximación a un modelo para Pymes exportadoras. Revista Colombiana de Ciencias Administrativas 2(1), pp. 62 - 77 Recuperado a partir de: http://cipres.sanmateo.edu.co/index.php/rcca

\section{Economía circular, aproximación a un modelo para Pymes exportadoras}

\section{Resumen:}

El objetivo del artículo es realizar una aproximación a un modelo que pueda ser usado por las pequeñas y medianas empresas (Pymes) que buscan oportunidades de optimizar el uso de materias primas, el diseño de eco-productos y ampliar las políticas internas que cambien pensamientos de compartir en vez de adquirir. Asimismo, transformar modelos tradicionales de consumo lineal e ingresar a una economía que aprovecha todos los recursos, manteniéndolos útiles por mayor tiempo. El método desarrollado en el artículo fue la búsqueda de información a nivel nacional e internacional, es decir, qué modelos se estaban aplicando y cómo se podrían ajustar a las Pymes colombianas. En ese sentido, el resultado de mayor importancia fue generalizar un método para las eco-industrias, basadas en la aplicación de tecnologías amigables con el medio ambiente y a la sustentabilidad.

Palabras clave: economía circular; reciclaje; ciclo técnico; ecodiseño; Pymes exportadoras.

\section{Circular economy, an approximation to a model for exporting SMES}

\section{Abstract:}

The objective of the article is to make an approximation to a model that can be used by SMEs looking for opportunities to modify the use of raw materials, the design of eco-products and to expand internal policies that change thoughts of sharing instead of acquiring. It also transform traditional 
linear consumption models and enter an economy that takes advantage of all resources, keeping useful supplies for longer. The developed method of the article was the search for information at a national and international level, the models that were being applied and how these were adjusted to Colombian SMEs. The most important result was to generalize a method for eco-industries that are based on the application of environmentally friendly technologies and sustainability.

Keywords: circular economy; recycling; technical cycle; eco-design; exporting SMEs

\section{Introducción}

Los seres humanos como seres inteligentes deben transformar sus pensamientos económicos entorno al planeta y sus recursos; además, utilizar toda la inteligencia evolutiva, financiera, cultural, humanística y procedimental para hacer una convivencia más fácil entre la naturaleza y los seres humanos; asimismo, aprender a reutilizar los recursos, no solo que puede ser aprovechados por una sola vez. Al menos a nivel teórico existe un cierto consenso sobre la necesidad de practicar un consumo sostenible (González, 2016).

Así, el presente artículo tiene como antecedentes los preceptos de Ellen MacArthur, navegante y filántropa, fundadora en el 2010 de Ellen MacArthur Foundation, una organización benéfica que trabaja con empresas y educación para acelerar la transición a una economía circular.

La fundación EllenMacarthur investiga, desde otras ópticas, los modelos actuales de la industria extractiva, que genera residuos y basa sus políticas fundacionales en una economía circular, que busca redefinir el crecimiento. A su vez, se enfoca en beneficios positivos para toda la sociedad. Implica desacoplar gradualmente la actividad económica del consumo de recursos finitos y diseñar residuos fuera del sistema.

Respaldado por una transición a fuentes de energía renovables, el modelo circular crea capital económico, natural y social. Los tres (3) principios de dicha fundación son diseñar sin generar residuos, ni contaminación; mantener los productos y materiales en uso y regenerar los sistemas naturales. La economía circular es una posibilidad de ayudar al planeta. 
Para esto deben cambiar los conceptos de economía lineal como se muestra en la Figura 1. (Cadena de abastecimiento lineal), a una cadena de abastecimiento circular, como lo muestra la Figura 2.

Figura 1. Cadena de abastecimiento lineal.

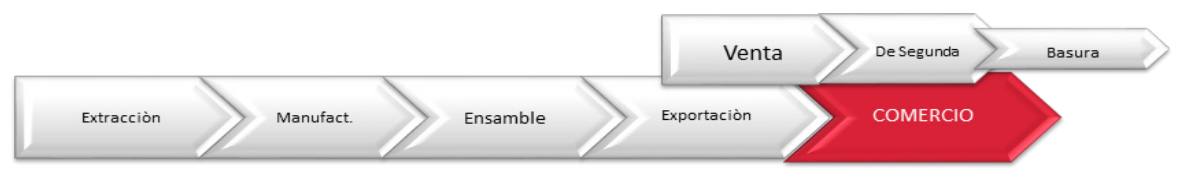

Fuente: Elaboración propia, a partir de Ellen Macarthur Foundation, s.f.

Figura 2. Cadena de abastecimiento circular

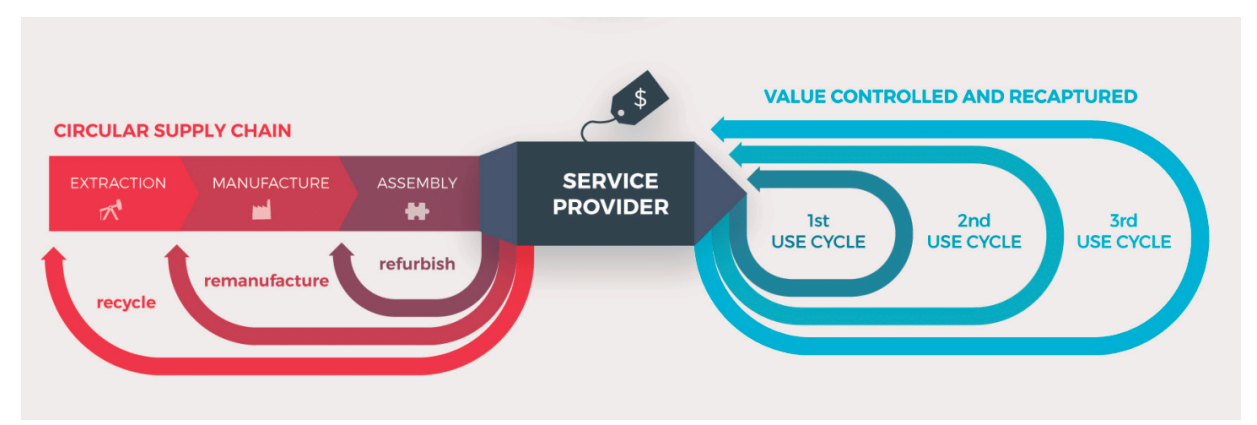

Fuente: Ellen Macarthur Foundation, "Service provider", s.f.

Más allá de un asunto económico, el tema abordado en el artículo se fundamenta en un asunto social; todos viven en el planeta y los cambios se deben iniciar con transformaciones en las unidades económicas más pequeñas (como las Pymes) para generar los avances necesarios en los procesos productivos de mayor envergadura. ¿Será que una aproximación a un modelo de economía circular eficiente ayudaría a iniciar esas transformaciones?

En este sentido, el objetivo principal es poder aproximarse a un modelo de economía circular general para la aplicación en los procesos de las Pymes, para alcanzar un estado del arte superior al actual donde tengan en cuenta no solo las bases económicas de los procesos, sino también bases medioambientales. Lo anterior, puesto que el planeta está exigiendo desde hace años un cambio en los modelos de negocios donde 
se le involucre, no solo como un proveedor de recursos, en una economía irresponsablemente lineal; sino ayudándolo y ayudándonos para mejorar la convivencia y el clima.

\section{Marco teórico}

\section{Los cambios requeridos para una economía sustentable.}

Los modelos de producción actuales se basan en una economía lineal de consumo de recursos principalmente modelado en extraer, fabricar, consumir y eliminar. Dicho modelo actualmente ocasiona el agotamiento de los recursos naturales (Porcelli y Martínez, 2018). Estamos agotando los recursos naturales con mayor rapidez, comprometiendo la futura seguridad de los recursos de la humanidad; dirigiéndonos a un cambio climático y a fenómenos meteorológicos extremos con mayor frecuencia y en zonas nunca vistos.

Este exceso puede ser temporal, la raza humana tendrá o deberá funcionar en los límites ecológicos de la tierra, se ajustará los seres humanos a estos límites ya sea por desastre o diseño. De esta manera "las empresas y los países que entienden y gestionan su realidad consistente con nuestro único planeta están en una posición mucho mejor para tener éxito en el siglo XXI", escribe Mathis Wackernagel (2019) de Global Footprint Network.

Los recursos como el aire, la tierra y el agua en estado limpio (que ofrece el planeta para el 2019) se agotaron el último día de julio de ese año; se está provocando una disminución en la regeneración de los recursos naturales y el planeta busca equilibrar este desfase natural. Se deben comprometer a mover esa fecha a los más cercano al final de cada año, con iniciativas como "\#movethedate", que ayudan a correr esta fecha; son iniciativas sencillas, con frecuencia financieramente ventajosas, encontrándose oportunidades en áreas como ciudades, energía, alimentos, población y planeta. Las empresas y gobiernos pueden optar por regenerar, compartir, optimizar, bucle, virtualizar e intercambiar para migrar al modelo circular (Pardo, 2018).

\section{Diferencias entre economía lineal y economía circular.}

El consumo desmedido de la economía lineal, desarrollado en su patrón de tomar, fabricar, consumir, eliminar (take-make-dispose) (Hartley, van Santen y Kirchherr, 2020) basado en ideas de recursos ilimitados, siendo realmente recursos no renovables, ha llevado a varios países a extralimitar 
su capacidad de consumo, como se observa en la Figura 3 (Hectáreas globales por persona).

Este mapa muestra cuáles países consumen más recursos naturales en 2014, en la escala de hectáreas globales por persona (hag), unidad utilizada para medir el impacto del consumo humano en el mundo.

Figura 3. Hectáreas globales por persona (hag)

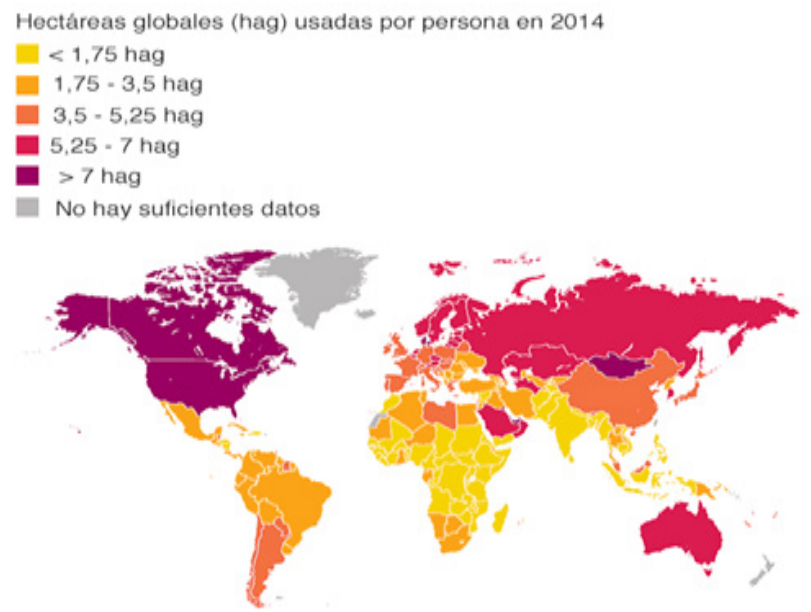

Fuente: Grooten y Almond (Eds.) "Informe Planeta Vivo", 2018

Este modelo acrecienta el consumo y la pérdida de ecosistemas, genera una degradación al medio ambiente y muestra fragilidad en el sistema económico; no lo perpetúa, sino que tendría un final al acabar los recursos. En contrapartida, el modelo de economía circular, planteado desde los años 1970, e intensificado sus análisis y estudios desde 1990, propone un modelo para que los recursos se mantengan en uso por mayor tiempo. Genera nuevas formas de producción y consumo, nuevas áreas de trabajo e investigación; fuerza a la economía a extraer todo lo valioso de cada unidad de consumo hasta su último átomo sin tomar o consumir nuevos recursos.

Este modelo enfatiza en la optimización de toda la cadena de valor, para realizar la conversión de los residuos en activos. Esta última parte es el mayor cambio generado por la economía circular; transforma el pensamiento de desechos a activos que deben ser valorizados en las compañías (Ayala, Llanos y Oyarzun, 2017). 


\section{Economía circular. Un modelo para ayudar.}

En el año 1992 la cumbre de Río (Brasil), constituyó un momento sin precedentes en el estudio del medio ambiente dentro del ámbito económicoempresarial. En este evento se amplió el concepto de "desarrollo sostenible", ratificado por 180 países (Balboa y Somonte, 2014). Stahel (2010), planteó la visión de una economía en bucles y el consecuente impacto en la creación de empleo, competitividad económica, ahorro de recursos y prevención de residuos.

La economía circular fue concebida como un concepto económico donde interrelacionan la sostenibilidad, el crecimiento económico y la minimización de la generación de residuos. La economía circular busca que los productos sean ensamblados y desensamblados, no generar residuos y que las partes de los productos después de usados sean materia prima para otros procesos. La economía circular ha desarrollado diferentes principios como:

Eco-concepción: En el diseño de los productos se cuidan los impactos ambientales del mismo durante su ciclo de vida útil y su disposición final de su uso.

Ecología industrial: Considera los ecosistemas industriales como una solución para la reutilización de sus residuos, optimizando los flujos de los recursos como agua y energía.

Economía de la funcionalidad: Privilegia los servicios por encima de los productos, ver los productos como servicios donde se deben realizar una devolución luego de usarlo en los procesos industriales o personales.

El uso de los productos bajo las tres (3) R (Reutilizar, Reciclar, Reparar): La economía circular fue planteada como un modelo que puede autosustentarse; sostenido en los ciclos biológicos y técnicos desarrollando un continuo uso de los recursos basado en tres (3) principios.

Preservación y mejoramiento del capital natural: A través del control de stocks finitos y equilibrando el flujo de los recursos renovables.

Optimización en el uso de los recursos: Circulando productos, componentes y materiales en el nivel más alto de utilidad, tanto en el ciclo técnico como en lo biológico. 
Fomentación de la eficacia del sistema: A través de la obtención de patentes y la eliminación de externalidades negativas (Tena y Khalilova, 2016; Caicedo, 2017).

Sin embargo, los seres humanos siempre generamos residuos. El modelo no es perfecto en la generación de residuos, pero podemos minimizarlos y crear un menor impacto para las generaciones futuras (Alier, 2003).

\section{Estado del arte de algunos modelos de economía circular en el mundo}

Tabla 1. Modelos de economía circular

\begin{tabular}{|c|c|c|}
\hline MODELO & PRINCIPIO & EJEMPLOS \\
\hline Suministros circulares & $\begin{array}{l}\text { Uso de energías renovables o } \\
\text { materiales reciclables en lugar } \\
\text { de recursos no renovables }\end{array}$ & $\begin{array}{l}\text { * Ecovatite Design: Compañía de biomateriales enfocada en el desarrollo de materiales } \\
\text { innovadores a partir de procesos de crecimiento natural. } \\
\text { * Lanzatech: Empresa de Nueva Zelanda que utiliza microorganismos para convertir el } \\
\text { monóxido de carbono (CO) en etanol y precursores plásticos. } \\
\text { * Aquazone: Compañáa finlandesa que ha desarrollado un método para convertir las aguas } \\
\text { residuales en fertilizantes. El agua se puede usar para el riego o se puede reciclar más en el } \\
\text { agua potable. }\end{array}$ \\
\hline Recuperación de recursos & $\begin{array}{l}\text { Obtención de energía o recursos } \\
\text { a partir de los residuos o del } \\
\text { deshecho }\end{array}$ & $\begin{array}{l}\text { * Adidas: En } 2016 \text { lanzó su primera línea de zapatos producidos con material Parley Ocean } \\
\text { Plastic }{ }^{\mathrm{M}} \text {. Fue obtenido reciclando los residuos plásticos que se encuentran en los mares y } \\
\text { océanos. Cada par utiliza un promedio de } 11 \text { botellas de plásticos, Adidas anunció que se } \\
\text { habían vendido más de } 1 \text { millón de pares de zapatos. } \\
\text { * Recompute: Empresa que pone en venta ordenadores hechos con productos ecológicos y } \\
\text { materiales de cartón reciclado. } \\
\text { * Newlight Technologies: Fundada en el } 2003 \text {, ha desarrollado una tecnología de captura de } \\
\text { carbono que extrae carbono de los gases de efecto invernadero y lo utiliza para producir un } \\
\text { material bioplástico llamado AirCarbon }{ }^{\mathrm{TM}} \text {. Es un material natural que puede igualar el } \\
\text { rendimiento de los plásticos basados en petróleo. }\end{array}$ \\
\hline $\begin{array}{l}\text { Prolongación de la vida } \\
\text { útil del producto }\end{array}$ & $\begin{array}{l}\text { Actualizar, reparar y revender } \\
\text { para que duren más los } \\
\text { productos }\end{array}$ & $\begin{array}{l}\text { * Wallapop: Nació en } 2013 \text { con la aspiración de hacer un mundo colaborativo y más } \\
\text { sostenible, lanzando un mercado virtual. Hoy cuenta con más de } 40 \text { millones de usuarios y } \\
100.000 .000 \text { de productos. } \\
\text { * Percentil: Star-up, en cinco (5) años ha creado una plataforma de prendas de segunda mano } \\
\text { con ventas anuales a millón de prendas y con base en Alemania, Holanda, Francia y España. } \\
\text { * iFixit: Con su visión de reparar el mundo, cuenta con una plataforma con más de } 50.000 \\
\text { guías para reparar cualquier dispositivo. }\end{array}$ \\
\hline $\begin{array}{l}\text { Uso compartido de } \\
\text { plataformas }\end{array}$ & $\begin{array}{l}\text { Fomentar la colaboración entre } \\
\text { usuarios para que compartan el } \\
\text { uso de productos }\end{array}$ & $\begin{array}{l}\text { * Farmidable: Empresa que pone en contactos a agricultores, ganaderos y/o pequeños } \\
\text { productores con consumidores, sin intermediarios. Actualmente tiene base en Madrid y } \\
\text { Barcelona. } \\
\text { * Tetellus: plataforma colaborativa para aprender y enseñar el español a través de video } \\
\text { cursos gratuitos. } \\
\text { * Plan\&Go: Aplicación que pone en contacto a gente con los mismos intereses y aficiones } \\
\text { para que compartan planes y los gastos derivados de los mismos. Cuenta con mas de } 40.000 \\
\text { usuarios en España. }\end{array}$ \\
\hline Producto como servicio & $\begin{array}{l}\text { Los clientes bajo este modelo } \\
\text { "alquilan" el producto, lo usan y } \\
\text { lo devuelve }\end{array}$ & $\begin{array}{l}\text { *Drivy: Permite alquilar tu vehículo cuando no lo estás utilizando. Se ha convertido en el } \\
\text { servicio de autos compartidos más grande de Europa. } \\
\text { *ParkFy: Plataforma que conecta propietarios de plazas de garaje particulares con } \\
\text { conductores en necesidad de aparcamiento. Presente en cinco (5) ciudades españolas y con } \\
\text { más de } 5000 \text { operaciones realizadas. } \\
\text { * Splacer: Star-up israelí creada en } 2014 \text { en Tel Aviv ofrece a los usuarios, a través de una } \\
\text { plataforma online, una lista de locales (casas, galerías y oficinas desocupadas) a disposición } \\
\text { para realizar todo tipo de celebración, eventos o simplemente workshop de trabajo. Por ahora } \\
\text { unicamente actúa en Israel y Estados Unidos. }\end{array}$ \\
\hline
\end{tabular}

Fuente: Delgado, "Tendencias \& Innovación", 2019. 


\section{Colombia y la economía circular}

El Gobierno colombiano lanzó la Estrategia Nacional de Economía Circular, mediante el Ministerio del Medio Ambiente y Desarrollo sostenible. Es una estrategia de política nacional, debe enfocarse de arriba abajo, y su implementación se estructura siguiendo tanto un enfoque horizontal como vertical (Yong, 2007). Busca promover la innovación y la generación de valor de los sistemas productivos y el consumo; con medidas como la optimización, intercambio, compartir, reciclaje, regeneración de materiales, la protección y mejor uso del agua y la energía. La estrategia busca reducir la carga a los rellenos sanitarios de un 30\% de los municipios del país pues estos tienen una vida útil máxima para los próximos cinco (5) años.

La política de producir conservando y conservar produciendo buscar incentivar a las empresas, la cadena de valor y los consumidores para repensar el uso de los recursos; transformar los modelos de negocios y optimizar el consumo. Lo anterior, en un marco de responsabilidad del sistema de gestión de residuos, eficiencia del uso de materiales y cambios en los estilos de vida de los ciudadanos, enfocados en la sostenibilidad (Nava y Abreu, 2015).

La estrategia está diseñada en seis (6) líneas de acción. El Gobierno, con sus ministerios y viceministerios, los gremios del sector productivo y el sector académico, están involucrados en un trabajo articulado. Lo que busca el Ministerio de Ambiente y Desarrollo Sostenible es crear las sinergias para generar oportunidades que brinda una economía circular. Las seis (6) líneas de acción están definidas como: Fuentes y aprovechamiento de energía, ciclo de vida del agua, materiales y productos industriales, materiales de empaque envase, optimización y aprovechamiento de la biomasa, por último, gestión de materiales en centros urbanos.

Cada una de las líneas de acción maneja objetivos a corto y largo plazo. Convocan a los participantes a transformar sus modelos tradicionales en circulares; asimismo, proponen mecanismos para fomentar habilitadores de economía circular. 


\section{Iniciativas privadas en Colombia.}

Asociaciones de industriales en Colombia han iniciado actividades para obtener benéficos de la economía circular. Han desarrollado iniciativas como la visión 30/30; más de 100 empresas en 19 sectores productivos buscan la gestión de sus envases y empaques con acciones de reciclaje, reúso y reducción.

Otra iniciativa la constituye el Grupo Retorna, proyecto especial del posconsumo de la Asociación Nacional de Industriales (ANDI); implementa el principio de Responsabilidad Extendida del Productor (REP). Forma alianzas como Red Verde donde, a nombre de las empresas, miembros del colectivo de la administración, operación y financiación del sistema de recolección selectiva y gestión ambiental, se encargan de los electrodomésticos cuando han cumplido su ciclo de vida y son descartados por los consumidores.

También, otras alianzas del Grupo Retorna como "Cierra el Ciclo" maneja el uso de envases y empaques de insecticidas domésticos. Asimismo, "Pilas con el ambiente" que coloca puntos de recolección de pilas usadas para dar disposición adecuada y que no lleguen a rellenos sanitarios ni a fuentes de agua. Por su parte, "Ecocomputo" recoge y administra los equipos de cómputos y periféricos desechados; "Recoenergy", está encargada de las baterías de automóviles y motos. Por último, "Ruedaverde" se basa en la recolección y disposición de llantas usadas.

Estas alianzas permiten introducir, al ciclo técnico de la economía circular, materias primas sin usar y nuevos recursos naturales. Buscan garantizar modalidades de consumo y producción sostenible, reduciendo la intensidad del uso de materiales y aprovechando las oportunidades de reciclaje y reutilización.

\section{Metodología}

La realización de esta investigación es de tipo cualitativa, a través de la realización de la observación externa o no participante, mediante las técnicas de búsqueda de ventanas de información desde los años 2010 en adelante. Lo anterior, principalmente, en bases como ScienceDirect, ResearchGate, Wiley Online Library Scielo; así como los ministerios de medio ambiente de América Latina. La investigación se desarrolló en cuatro (4) fases consecutivas relacionadas a continuación: 
-Preparatoria: Fase donde se planifica las actividades que se ejecutarán en las fases posteriores, preguntas orientadoras, enfoque y tipos de investigación. Además, se detalló el objetivo de la tutoría - investigación y los determinadores del documento final.

-Búsqueda de ventana de información: Consiste en recopilar información necesaria para llegar a una o varias conclusiones o soluciones de la pregunta inicial. Se basa en la búsqueda de matrices de datos y recolección de documentación de expertos en relación con la economía circular, la sustentabilidad y las organizaciones enfocadas en los temas de preservación del medio ambiente y la economía.

-Análisis de la información: Comprobar y valorar la información recolectada, evaluar lo encontrado, concatenar las ideas principales de la documentación y buscar la validez de las acciones, datos e información.

-Aproximación a un modelo: Setrata de diseñar una aproximación valida de modelos aplicables para Pymes exportadora; de fácil implementación en sus estrategias de economía circular, aprovechando las ventajas de las propuestas entregadas por el gobierno.

\section{Resultados}

Las Pymes, por su naturaleza, asimilan y adaptan fácilmente las tecnologías de diversos tipos; producen innovación en sus procesos con mayor rapidez y resultados con mayor impacto. Las Pymes mantienen gran flexibilidad para adecuarse a las normativas del entorno; buscan beneficios, rentabilidad y reputación, al asumir modelos disruptivos como los planteados por la economía circular.

Para alcanzar estas propuestas las Pymes deben buscar estrategias para ingresar a mercados rentables como los europeos. Estas estrategias pueden ser la cooperación con otras Pymes (o asociaciones industriales) que permitan transformar sus procesos productivos, siendo evolutivos hacia un ambiente sustentable.

\section{Aproximación de un modelo de economía circular para Pymes exportadoras.}

Los empresarios deben redefinir su perspectiva de creación de valor colocando el planeta y a los clientes en el centro de sus procesos, además 
redefinir sus modelos de negocios. Las empresas deben recapacitar en la sustentabilidad del negocio como eje fundamental de la economía circular.

En este sentido, se inicia con las Pymes (sobre todo las exportadoras); sus productos tienen mayor exposición en el ámbito internacional. Además, la Organización para la Cooperación y el Desarrollo Económico (OCDE), en la cual Colombia ingresó en el 2018, tiene entre sus políticas trabajar con los gobiernos en temas como los factores que impulsan el cambio económico, social y ambiental. Es responsabilidad de Colombia hacer la transición a estas buenas prácticas bajo los marcos de planeación (Rodríguez, 2014).

Conforme con lo postulado en el lanzamiento del proceso de adhesión la membresía de OCDE permitirá al país evaluar y comparar las políticas y marcos institucionales. Lo anterior, con las mejores prácticas internacionales en diversidad de sectores como el fiscal, salud, medio ambiente, educación, combate a la pobreza, entre otros (Rodríguez, 2014).

\section{Las entradas del modelo.}

Análisis para un rediseño de producto basados en ecodiseño; las Pymes deben considerar sus productos y modelos productivos, basados en el ecodiseño, permitiendo que los usuarios accedan a servicios en lugar de productos; buscando aproximaciones, ajustes o reconversión de sus procesos en los modelos expuestos en la Tabla 1 (Modelos de economía circular y culturas de alquiler, rendimiento y el compartir). Así, reducen los residuos en el diseño.

Prospección, búsqueda y negociación con proveedores sustentables, es decir, transformar los procesos de compras de materias primas de ciclos biológicos a compras de ciclos técnicos. Asimismo, buscar alternativas de materiales de envases y empaques basados en estas premisas.

Conversión de los procesos productivos a modelos sustentable. Esto es aprender a producir de forma amigable con el planeta, con procesos de recirculación de energía, agua y con el consumo de materias primas de ciclos técnicos; pensar como pertenecientes a sistemas relacionados y vinculados entre sí. Por otro lado, rediseñar los empaques con materias primas reusadas, reutilizadas o que las usadas sirvan para ingresar a ciclos técnicos de reciclaje. 
La economía circular propone cambiar los modelos de productos a servicios, desmaterializando la economía, para ser sostenibles y mejorar el compartir que comprar. Piensa que los productos o servicios circulen más tiempo en los procesos técnicos con modelos de reutilización, luego de reciclaje y, por último, de desensambles, antes de entrar de nuevo a los ciclos de materias primas (como se muestran en la Figura 4).

Figura 4. Aproximación a un modelo

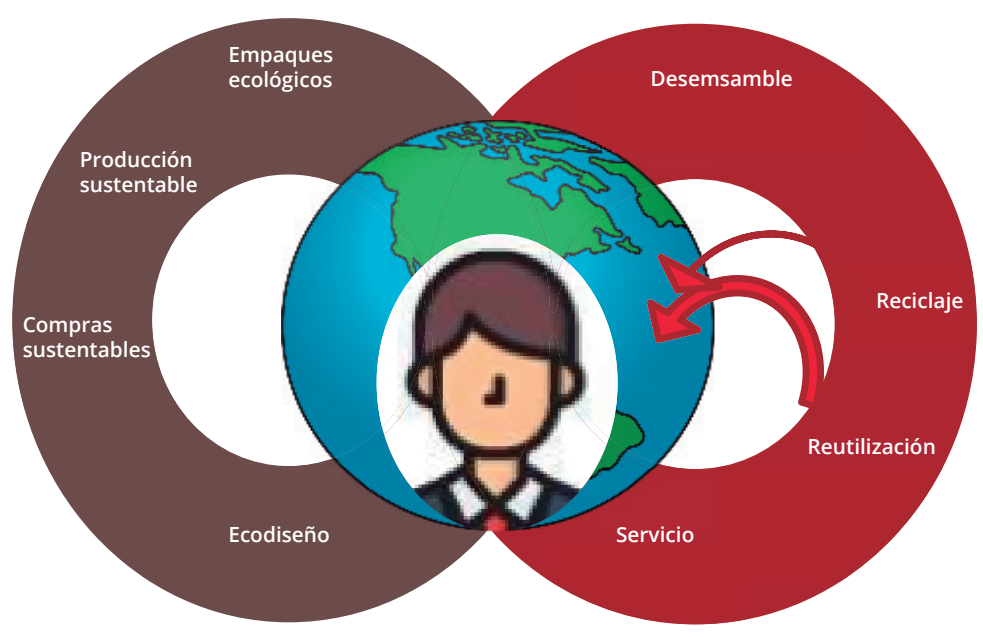

Fuente: Elaboración propia.

\section{Salida del modelo.}

Se esperaría que los resultados de la aplicación de alguna de las entradas del modelo generen productos o servicios sustentables, auditables y certificables con las buenas prácticas ambientales, establecidas en el marco de la economía circular.

\section{Conclusiones}

Las Pymes, como motor de las economías de los países, son las empresas llamadas a liderar los cambios en sus procesos productivos para ingresar a modelos sustentables de economías circular (Cerda y Khalilova, 2016).

Como lo expresa Navarro, García y Vela (1999), los crecimientos económicos de las Pymes deben reflexionarse para que provengan de ingresos derivados de actividades circulares con menores costos de 
producción por la reducción de compra de insumos vírgenes; asimismo, la mayor utilización de insumos de ciclos técnicos de materias reusadas. Estos cambios en los insumos y productos propagan efectos indirectos en otros sectores de la economía incrementando los crecimientos totales de la misma.

Los modelos de consumo colaborativo son reconocidos como una de las mejores opciones disponibles en el lado del consumidor para pasar del actual modelo de negocio habitual a economía circular. Los modelos de colaboración (por ejemplo, compartir, intercambiar, prestar, comerciar, alquilar, regalar) se basan en una propiedad compartida entre múltiples consumidores (Ghisellini, Cialani y Ulgiati, 2016).

Para los componentes de la innovación de las Pymes, el objetivo es sustituir insumos unidireccionales o lineales por insumos circulares, basados en su diseño, generando logísticas inversas, con sistemas de respaldos. Además, estimulando nuevos procesos y ecosistemas industriales, basando este crecimiento en el uso de tecnologías, mano de obra especializada, eficiencias energéticas y con mayores impactos en las oportunidades económicas de las Pymes.

La aproximación a los modelos expuestos, y al modelo propuesto, pretenden iniciar una serie de estudios que busquen la aplicabilidad en diversos tipos de Pymes; ya sean exportadoras o del mercado nacional, que ayuden a evaluar y generar cambios en sus estructuras a mediano plazo. En este sentido, aportar a mejorar sus resultados, tanto en el plano productivo como el de responsabilidad social empresarial.

\section{Agradecimientos}

Los autores desean agradecer a las estudiantes de la Fundación Universitaria San Mateo Nelsy González y Angie Sánchez que apoyaron en la ejecución del proyecto de investigación y redacción de este artículo. 


\section{Referencias}

Alier, J. (2013). Conflictos ecológicos por extracción de recursos y por producción de residuos. Letras Verdes. Revista Latinoamericana de Estudios Socioambientales, (3), pp. 8-10.

Ayala, E., Llanos, M., y Oyarzun, A. (2017). Laboratorio de innovación como apoyo a la formación emprendedora estudiantes de ingeniería UNAP, caso práctico economía circular. Revista Universitaria Ruta, 19(2), pp. 48-57. Recuperado de: https://revistas.userena.cl/index.php/ruta/article/view/987

Balboa, C., y Somonte, M. (2014). Economía circular como marco para el ecodiseño: el modelo ECO-3. Informador técnico, 78(1), pp. 82-90.

Caicedo, C. (2017). Economía circularysu papel en el diseño e innovación sustentable [en línea]. Recuperado de http://ojseditorialumariana.com/ index.php/libroseditorialunimar/article/view/1154/1095.

Cerda, E. y Khalilova, A. (2016). Economía circular. Revista Empresa, medio ambiente y competición, 401, pp. 11-20.

Delgado, Emilio. (2019). Tendencias e Innovación. Economía Circular y sus modelos. https://obsbusiness.school/es/blog-investigacion/post-deembajadores/economia-circular-y-sus-modelos-de-negocio

Durán, G. (2019). Progresando hacia un modelo de economía circular. Revista economía y medio ambiente, pp. 211-215.

Ghisellini, P., Cialani, C. y Ulgiati, S. (2016). A review on circular economy: the expected transition to a balanced interplay of environmental and economic systems. Journal of Cleaner Production, (114), pp. 11-32.

González, L. (2016). ¿Del consumo sostenible a una economía circular? Revista CESCO de Derecho de Consumo, (17), pp. 179-191.

Grooten, M. y Almond, R. (Eds.). (2018). Informe Planeta Vivo - 2018: Apuntando más alto. Suiza: Worldwide Fund for Nature (WWF). Recuperado de: chttp://awsassets.wwf.es/downloads/informe_planeta_vivo_2018.pdf 
Hartley, K., van Santen, R., y Kirchherr, J. (2020). Policies for transitioning towards a circular economy: Expectations from the European Union (EU). Resources, Conservation \& Recycling, (155), pp. 1-10.

Nava, J. y Abreu, Y. (2015). Logística Verde y Economía Circular. International Journal of Good Conscience, 10(3), pp. 80-91.

Navarro, L., García, M., y Vela, M. (1999). El panorama actual de las pymes: ventajas y desventajas competitivas. ESIC MARKET, 107, pp. 161.178.

Pardo, M. (2018). Smart cities, economía circular y aprovechamiento de residuos sólidos en Bogotá. Colombia: Colegio de Estudios Superiores de Administración (CESA).

Porcelli, A. y Martínez, A. (2018). Análisis legislativo del paradigma de la economía circular. Revista Direito GV, 14(3), 1067-1105.

Rodríguez, G. (2014). La adhesión de Colombia a la OCDE y su efecto en las políticas públicas. Bogotá: Universidad Militar Nueva Granada.

Stahel, W. (2010). The performance economy. England: Springer.

Tena, E., \& Khalilova, A. (2016). Economía circular. Economía industrial, (401), pp. 11-20.

Wackernagel, Mathis (26 de junio de 2019). Earth Overshoot Day 2019 is July 29th, the earliest ever. Oakland, Ca, USA [en línea]. Recuperado de https://www.footprintnetwork.org/2019/06/26/press-release-june-2019earth-overshoot-day/

Yong, R. (2007). The circular economy in China. Journal of Material Cycles and Waste Management, (9), pp. 121-129. 